\title{
Rotenone Remarkably Attenuates Oxidative Stress, Inflammation, and Fibrosis in Chronic Obstructive Uropathy
}

\author{
Ying Sun, ${ }^{1,2,3,4}$ Yue Zhang, ${ }^{1,2,3}$ Daqiang Zhao, ${ }^{5}$ Guixia Ding, ${ }^{1,2,3}$ \\ Songming Huang, ${ }^{1,2,3}$ Aihua Zhang, ${ }^{1,2,3}$ and Zhanjun Jia ${ }^{1,2,3}$ \\ ${ }^{1}$ Nanjing Key Laboratory of Pediatrics, Nanjing Children's Hospital, Nanjing Medical University, Nanjing 210008, China \\ ${ }^{2}$ Department of Nephrology, Nanjing Children's Hospital, Nanjing Medical University, Nanjing 210008, China \\ ${ }^{3}$ Institute of Pediatrics, Nanjing Medical University, Nanjing, China \\ ${ }^{4}$ Division of Nephrology, Department of Medicine, New York University Langone Medical Center, New York, NY 10016, USA \\ ${ }^{5}$ Department of Renal Transplantation, The Third Affiliated Hospital, Sun Yat-sen University, 600 Tianhe Road, \\ Tianhe District, Guangzhou 510630, China
}

Correspondence should be addressed to Aihua Zhang; zhaihua@njmu.edu.cn and Zhanjun Jia; jiazj72@hotmail.com

Received 5 May 2014; Revised 30 June 2014; Accepted 10 July 2014; Published 22 July 2014

Academic Editor: Yung-Hsiang Chen

Copyright (C) 2014 Ying Sun et al. This is an open access article distributed under the Creative Commons Attribution License, which permits unrestricted use, distribution, and reproduction in any medium, provided the original work is properly cited.

\begin{abstract}
Mitochondrial abnormality has been shown in many kidney disease models. However, its role in the pathogenesis of chronic kidney diseases (CKDs) is still uncertain. In present study, a mitochondrial complex I inhibitor rotenone was applied to the mice subjected to unilateral ureteral obstruction (UUO). Following 7-days rotenone treatment, a remarkable attenuation of tubular injury was detected by PAS staining. In line with the improvement of kidney morphology, rotenone remarkably blunted fibrotic response as shown by downregulation of fibronectin (FN), plasminogen activator inhibitor-1 (PAI-1), collagen I, collagen III, and $\alpha$-SMA, paralleled with a substantial decrease of TGF- $\beta_{1}$. Meanwhile, the oxidative stress markers thiobarbituric acidreactive substances (TBARS) and heme oxygenase 1 (HO-1) and inflammatory markers TNF- $\alpha$, IL-1 $\beta$, and ICAM- 1 were markedly decreased. More importantly, the reduction of mitochondrial DNA copy number and mitochondrial NADH dehydrogenase subunit 1 (mtND1) expression in obstructed kidneys was moderately but significantly restored by rotenone, suggesting an amelioration of mitochondrial injury. Collectively, mitochondrial complex I inhibitor rotenone protected kidneys against obstructive injury possibly via inhibition of mitochondrial oxidative stress, inflammation, and fibrosis, suggesting an important role of mitochondrial dysfunction in the pathogenesis of obstructive kidney disease.
\end{abstract}

\section{Introduction}

Fibrosis is a common event of various forms of chronic kidney diseases (CKDs). The progression of renal fibrosis has been thought as a major pathological process leading to the progressive loss of renal function in CKDs [1-3]. Among those causative factors leading to the renal fibrosis, inflammation and oxidative stress were best characterized [4-7]. In the past decades, although numerous studies have been performed aiming to develop better strategies for treating the CKDs $[8,9]$, the therapeutic outcome is still unsatisfactory owing to the incomplete understanding of pathological mechanisms.
Interestingly, recent reports indicated an abnormal change of mitochondria in some CKD models including UUO [10-12] and 5/6 nephrectomy [13]. Mitochondria not only are the key source of energy production but also play important roles in mediating the signaling transduction, cell proliferation and the control of cell cycle, cell growth, and cell death $[14,15]$. The dysfunction of mitochondria causes ATP depletion, reactive oxygen species (ROS) overproduction, and release of proapoptotic factors like cytochrome $\mathrm{C}$ and mitochondrial DNA, which could result in the cell injury via oxidative damage of DNA and protein and apoptotic response and subsequent inflammation and fibrosis [16, 17]. In agreement with these notions, our previous study gave 
the evidence showing that mitochondrial dysfunction is an early event prior to the occurrence of renal fibrosis in mouse model with aldosterone infusion [18] and the intervention of mitochondrial dysfunction remarkably attenuated renal injury induced by chronic aldosterone infusion. In obstructed kidneys, the mitochondrial abnormality is also proven $[10$, 12 ], suggesting an involvement of mitochondrial dysfunction in the pathogenesis of obstructive kidney disease.

UUO is a well-established and widely used model in investigating the mechanisms and therapeutic strategies of renal fibrosis [19]. The mitochondrial abnormality in obstructed kidney might be a cause leading to the renal fibrosis or merely a secondary result of renal injury. To define the role of mitochondrial dysfunction in obstructive kidney injury, we treated the UUO mice with a mitochondrial complex I inhibitor rotenone to determine (1) whether inhibition of mitochondrial complex I can attenuate tubular injury and renal fibrosis in obstructive kidney disease and (2) whether mitochondrial complex I inhibition could affect the oxidative stress and inflammation in this particular model.

\section{Methods}

2.1. Animals. C57BL/6J mice were originally purchased from Jackson lab. This mouse colony was propagated at the Nanjing Medical University. In all studies, 3- to 4-month-old male mice were used. All mice were maintained under a $12: 12 \mathrm{~h}$ light-dark cycle (lights on at 6:00 a.m. and lights off at 6:00 p.m.). This study was approved by the Nanjing Medical University Institutional Animal Care and Use Committee.

\subsection{Establishment of UUO Mouse Model and Rotenone Treat-} ment. Unilateral ureter obstruction was induced as described previously [20]. Briefly, the left ureter was exposed and subsequently ligated with 6.0 silk through a small abdominal incision under the anesthesia with $2.0 \%$ isoflurane. The abdomen was closed in two layers. All mice received analgesia (subcutaneous injection of $50 \mu \mathrm{g} / \mathrm{kg}$ buprenorphine (Temgesic, Schering-Plough)) after the surgery. Following the surgery, the jelly diet with or without rotenone at a dose of $500 \mathrm{ppm}$ was given to the UUO mice. The sham control mice were treated with jelly diet without rotenone. After seven-day treatment of rotenone, mice ( $N=5$ per group) were sacrificed and the kidney tissues were harvested for the evaluation of gene and protein expressions and histological analysis.

2.3. Tubular Injury Score. Kidney tissues were fixed by direct immersion in $10 \%$ formalin for $16 \mathrm{~h}$. Following embedding in paraffin, $4 \mu \mathrm{m}$ sections were prepared and stained with periodic acid Schiff (PAS) and analyzed with light microscope. The percentage of tubular injury parameters of epithelial flattening, tubular dilatation, and brush border loss was estimated by a pathologist who was blind to the identity of the specimen using a 4-point scale in ten randomly chosen, nonoverlapping fields (200x magnification). Degree of injury was graded onto a scale from 0 to $4: 0=$ normal; $1=$ mild, involvement of less than $25 \%$ of the cortex; $2=$ moderate, involvement of 25 to $50 \%$ of the cortex; 3 = severe, involvement of 50 to $75 \%$ of the cortex; and $4=$ extensive damage involving $>75 \%$ of the cortex.

2.4. Immunohistochemistry. Kidneys were fixed with $10 \%$ formalin and embedded in paraffin. Kidney sections $(4 \mu \mathrm{m}$ thickness) were incubated in $3 \% \mathrm{H}_{2} \mathrm{O}_{2}$ for 15 minutes at room temperature to block endogenous peroxidase activity. After boiling in antigen retrieval solution $(1 \mathrm{mmol} / \mathrm{L}$ tris- $\mathrm{HCl}$, $0.1 \mathrm{mmol} / \mathrm{L}$ EDTA, $\mathrm{pH}=8.0$ ) for 15 minutes at high power in a microwave oven, the sections were incubated overnight at $4^{\circ} \mathrm{C}$ with rabbit anti-collagen I antibody (Cat number: sc8784, Santa Cruz). After washing with PBS, the secondary antibody was applied and the signal was visualized using an ABC kit (Santa Cruz Biotechnology).

2.5. Immunoblotting. The whole kidney was lysed and protein concentration was determined by Coomassie reagent. Proteins $(60 \mu \mathrm{g})$ from whole kidney lysates were denatured in boiling water for $10 \mathrm{~min}$, separated by SDS-polyacrylamide gel electrophoresis, and transferred onto nitrocellulose membranes. The blots were blocked overnight with 5\% nonfat dry milk in tris-buffered saline (TBS), followed by incubation for $1 \mathrm{~h}$ with rabbit anti-collagen I (Cat number: sc-8784, Santa Cruz), anti-fibronectin (Cat number: sc-9068, Santa Cruz), anti- $\alpha$-smooth muscle actin (Cat number: sc-32251, Santa Cruz), or anti-HO-1 (Cat number: ab13248, Abcam) at a dilution of $1: 1000$. After being washed with TBS, blots were incubated with a goat anti-horseradish peroxidaseconjugated secondary antibody (1:1000 dilution) and visualized with ECL kits (Amersham, Piscataway, NJ, USA).

2.6. $q R T-P C R$. Total RNA isolation and reverse transcription were performed as previously described [21]. Total DNA from kidney was isolated using the DNeasy Tissue Kit (Invitrogen, Carlsbad, CA). The mRNA and mtDNA copy numbers were detected by qRT-PCR. Oligonucleotides were designed using Primer3 software (available at http://frodo.wi.mit.edu/ primer3/) and the sequences are shown in Table 1. qRT-PCR amplification was performed using the SYBR Green Master Mix (Applied Biosystems, Warrington, UK) and the PRISM 7500 Real-Time PCR Detection System (Applied Biosystems, Foster City, CA, USA). Cycling conditions were $95^{\circ} \mathrm{C}$ for $10 \mathrm{~min}$, followed by 40 repeats of $95^{\circ} \mathrm{C}$ for $15 \mathrm{~s}$ and $60^{\circ} \mathrm{C}$ for $1 \mathrm{~min}$.

2.7. Measurement of Thiobarbituric Acid-Reactive Substances. The measurement of plasma thiobarbituric acid-reactive substances (TBARS) was based on the formation of malondialdehyde by using a commercially available TBARS Assay kit (Cat number: 10009055; Cayman Chemical) according to the manufacturer's instructions.

2.8. Enzyme Immunoassay. The kidney tissue was homogenized in phosphate-buffered saline and then centrifuged for $5 \mathrm{~min}$ at 10,000 r.p.m. The supernatant was diluted 1:50 with enzyme immunoassay buffer. Concentrations of TGF$\beta_{1}$ were determined by enzyme immunoassay according to 

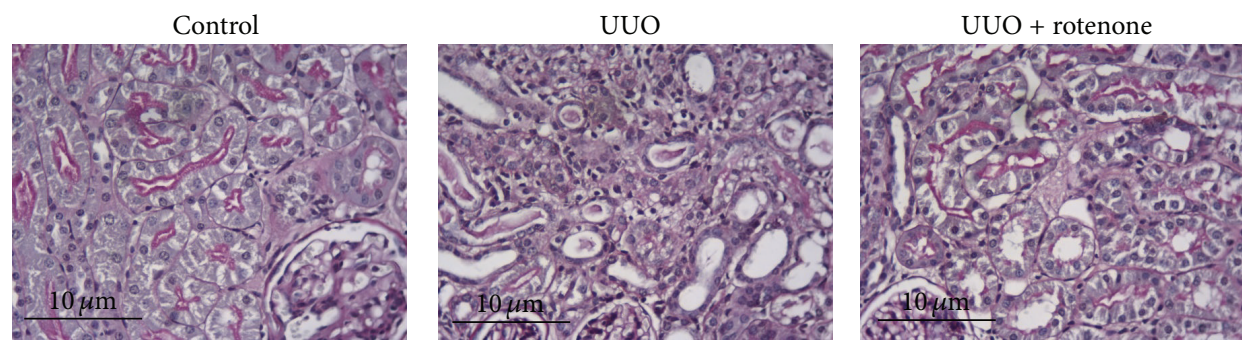

(a)

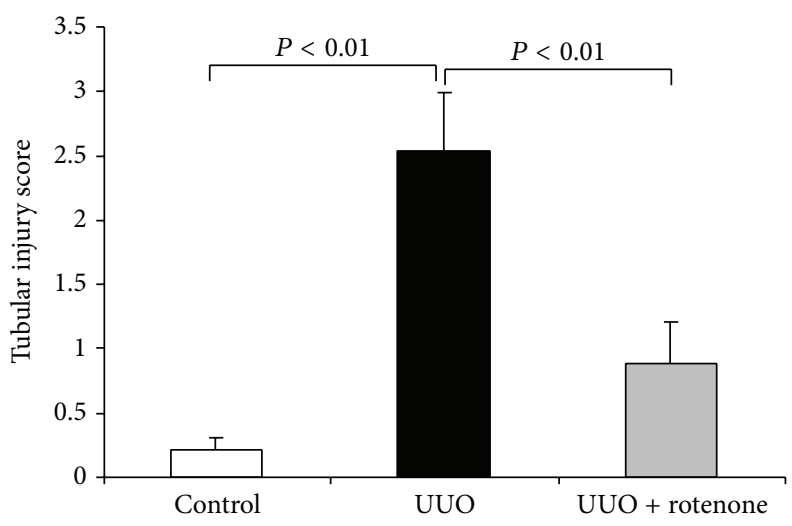

(b)

FIGURE 1: Rotenone treatment attenuated renal tubular injury. (a) Representative images of renal PAS staining in control and UUO mice with or without rotenone treatment. (b) Score of tubular injury. $N=5$ in each group. Data are means \pm SE.

manufacturer's instructions (Cat number: ab119557, Abcam). The kidney content of TNF- $\alpha$ and IL- $1 \beta$ was measured using the ELISA kits (TNF- $\alpha$ : Cat number: 559732 , BD OptEIA, BD Bioscience; IL-1 $\beta$ : Cat number: ab100704, Abcam).

2.9. Statistical Analysis. All values are presented as mean \pm SE. Statistical analysis was performed using Student's $t$-test or two-way ANOVA. Differences were considered to be significant when $P<0.05$.

\section{Results}

3.1. Effect of Rotenone Treatment on Renal Structural Change in Obstructed Kidneys. Following 7-day ureteral obstruction, the PAS staining indicated marked tubular structure damage as shown by the loss of brush border, epithelial cell atrophy and flattening, and tubular lumen dilation (Figure 1(a)). Strikingly, 7-day rotenone treatment remarkably attenuated all these morphological abnormalities (Figure 1(a)). Tubular injury score analysis also demonstrated a robust improvement of tubular injury as shown by Figure 1(b).

\subsection{Effect of Rotenone Treatment on the Oxidative Stress Level} in Obstructed Kidneys. Dysfunctional mitochondria serve as important source of ROS production. By blockade of mitochondrial complex I, upregulation of oxidative stress marker HO-1 was significantly blocked in obstructed kidneys (Figures 3(a) and 3(b)). To further validate this antioxidative

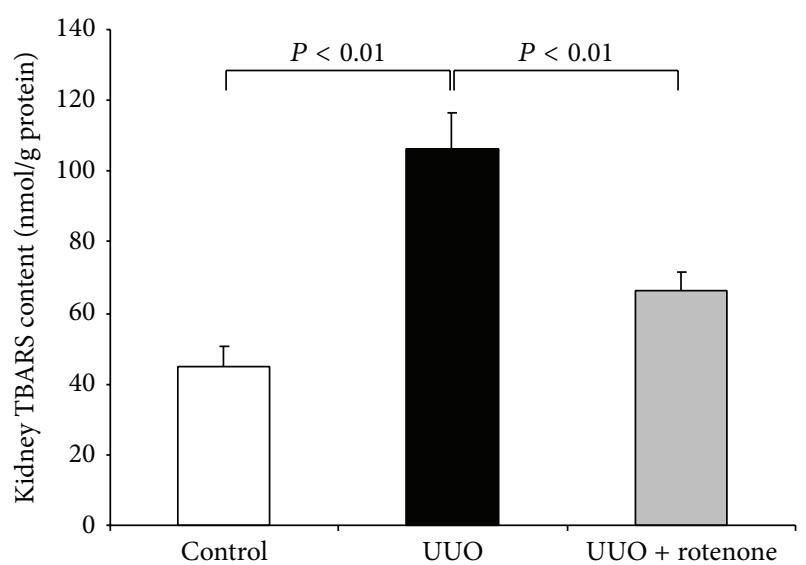

FIGURE 2: Rotenone treatment blocked the increase of TBARS in obstructed kidneys. Oxidative stress marker TBARS in kidneys was measured using a commercial kit. $N=5$ in each group. Data are means $\pm \mathrm{SE}$

effect of rotenone in this model, we examined TBARS level using a commercial kit. As expected, the increased TBARS content in obstructed kidneys was strikingly reduced by rotenone administration (Figure 2).

3.3. Effect of Rotenone Treatment on Inflammatory Response in Obstructed Kidneys. UUO is also an inflammatory kidney disease model with remarkably augmented inflammation 
TABLE 1: Sequences of primers for real-time PCR.

\begin{tabular}{|c|c|}
\hline Gene & Primer sequence \\
\hline \multirow{2}{*}{ GAPDH } & $5^{\prime}$-gtcttcactaccatggagaagg $-3^{\prime}$ \\
\hline & $5^{\prime}$-tcatggatgaccttggccag- $3^{\prime}$ \\
\hline \multirow{2}{*}{ TNF- $\alpha$} & $5^{\prime}$-tccccaaagggatgagaag- $3^{\prime}$ \\
\hline & $5^{\prime}$-cacttggtggtttgctacga- $3^{\prime}$ \\
\hline \multirow{2}{*}{ IL-1 $\beta$} & $5^{\prime}$-actgtgaaatgccaccttttg- $3^{\prime}$ \\
\hline & $5^{\prime}$-tgttgatgtgctgctgtgag- $3^{\prime}$ \\
\hline \multirow{2}{*}{ ICAM-1 } & $5^{\prime}$-cgcttccgctaccatcac- $3^{\prime}$ \\
\hline & $5^{\prime}$-ggcggctcagtatctcctc- $3^{\prime}$ \\
\hline \multirow{2}{*}{ PAI-1 } & $5^{\prime}$-cacgctacttcctcctcaag- $3^{\prime}$ \\
\hline & $5^{\prime}$-ctctgtcttcatcagctggc $-3^{\prime}$ \\
\hline \multirow{2}{*}{ FN } & $5^{\prime}$-cgtggagcaagaaggacaa- $3^{\prime}$ \\
\hline & $5^{\prime}$-gtgagtctgcggttggtaaa- $3^{\prime}$ \\
\hline \multirow{2}{*}{ Collagen I } & $5^{\prime}-$ ccggctcctgctcctctt- $3^{\prime}$ \\
\hline & $5^{\prime}$-ttgcacgtcatcgcacac- $3^{\prime}$ \\
\hline \multirow{2}{*}{ Collagen III } & $5^{\prime}$-tggtttcttctcacccttctt- $3^{\prime}$ \\
\hline & $5^{\prime}$-caaatgggatctctgggttg- $3^{\prime}$ \\
\hline \multirow{2}{*}{ TGF- $\beta_{1}$} & $5^{\prime}$-tacgcctgagtggctgtctt- $3^{\prime}$ \\
\hline & $5^{\prime}$-cgtggagtttgttatctttgct- $3^{\prime}$ \\
\hline \multirow{2}{*}{ mtDNA } & $5^{\prime}$-atcctcccaggatttggaat- $3^{\prime}$ \\
\hline & $5^{\prime}$-accggtaggaattgcgataa- $3^{\prime}$ \\
\hline \multirow{2}{*}{ 18S rRNA } & $5^{\prime}$-ttcggaactgaggccatgatt- $3^{\prime}$ \\
\hline & $5^{\prime}$-tttcgctctggtccgtcttg- $3^{\prime}$ \\
\hline \multirow{2}{*}{ mtND1 } & $5^{\prime}$-aatcgccatagccttcctaacat- $3^{\prime}$ \\
\hline & $5^{\prime}$-ggcgtctgcaaatggttgtaa- $3^{\prime}$ \\
\hline
\end{tabular}

from both infiltrating cells and resident cells. By qRT-PCR, we found that the inflammatory markers TNF- $\alpha$, IL-1 $\beta$, and ICAM-1 were markedly elevated following 7-day ureteral obstruction, and such increments were robustly abolished or attenuated by rotenone administration (Figure 4(a)). By ELISA, we further confirmed the protein regulation of TNF$\alpha$ and IL- $1 \beta$ following rotenone treatment (Figures 4(b) and $4(\mathrm{c}))$.

3.4. Effect of Rotenone Treatment on the Fibrotic Markers in Obstructed Kidneys. Fibrosis is the known pathological phenomenon in UUO animal models. To evaluate the effect of rotenone treatment on the fibrosis in this model, we examined protein and mRNA expressions of cellular matrix components including collagen I, collagen III, FN, PAI1 , and the fibroblast cell marker $\alpha$-SMA. Strikingly, both protein (Figures 5(a)-5(c)) and mRNA (Figures 6(a) and 6(b)) expressions of these markers were robustly downregulated by rotenone treatment. These findings demonstrated an antifibrotic role of rotenone in chronic obstructive kidney disease.

3.5. Effect of Rotenone Treatment on TGF- $\beta_{1}$ Expression in Obstructed Kidneys. TGF- $\beta$ is a known profibrotic factor in mediating the fibrotic process in obstructive kidney disease. To evaluate rotenone effect on this important fibrotic factor in this UUO model, we examined mRNA and protein levels of

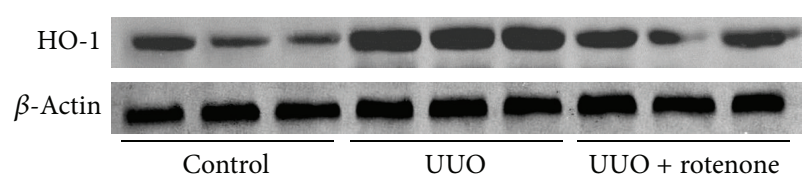

(a)

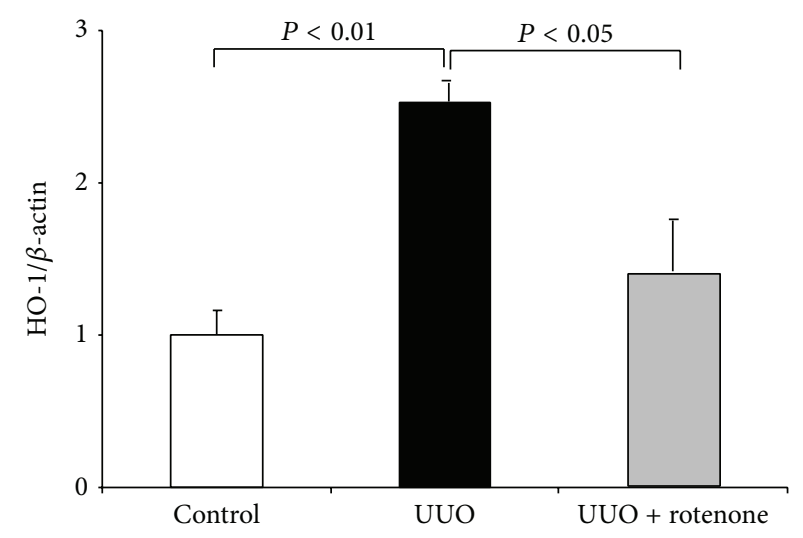

(b)

FIGURE 3: Rotenone treatment blunted HO-1 induction in obstructed kidneys. (a) Oxidative stress marker HO-1 was determined by Western blotting, and $\beta$-actin was used as loading control. (b) Densitometry of Western blots. $N=5$ in each group. Data are means \pm SE.

TGF- $\beta_{1}$ using qRT-PCR and ELISA, respectively. As shown by the data, both mRNA and protein levels of TGF- $\beta_{1}$ were significantly blunted by rotenone (Figures $7(a)$ and $7(b)$ ).

3.6. Effects of Rotenone Treatment on Mitochondrial DNA Copy Number and mtND1 Expression. To evaluate the mitochondrial abnormality, we measured mtDNA copy number and mtND1 expression. As shown by Figures 8(a) and 8(b), kidney obstruction remarkably reduced both indices, indicating a severe mitochondrial abnormality. After 7-day rotenone administration, reduction of mtDNA copy number and $\mathrm{mtND} 1$ expression was moderately but significantly restored (Figures $8(\mathrm{a})$ and $8(\mathrm{~b})$ ), suggesting amelioration of mitochondrial abnormality.

\section{Discussion}

The prevalence of CKD is rapidly rising with the increments of various insults leading to the kidney injury. Among those insults, diabetes and hypertension have become the major ones resulting in the CKD [22, 23]. In the kidneys of CKD patients, the inflammation, oxidative stress, and fibrosis are the common pathological manifestations and form a positive feedback loop to promote progressive renal injury and function loss [4-7]. In the past decades, although the researchers and nephrologists made substantial efforts in understanding the pathogenesis of CKDs, the current management strategies are still ineffective at stopping disease progression. This situation raised an urgent request to better understand the pathogenic mechanisms of CKDs. 


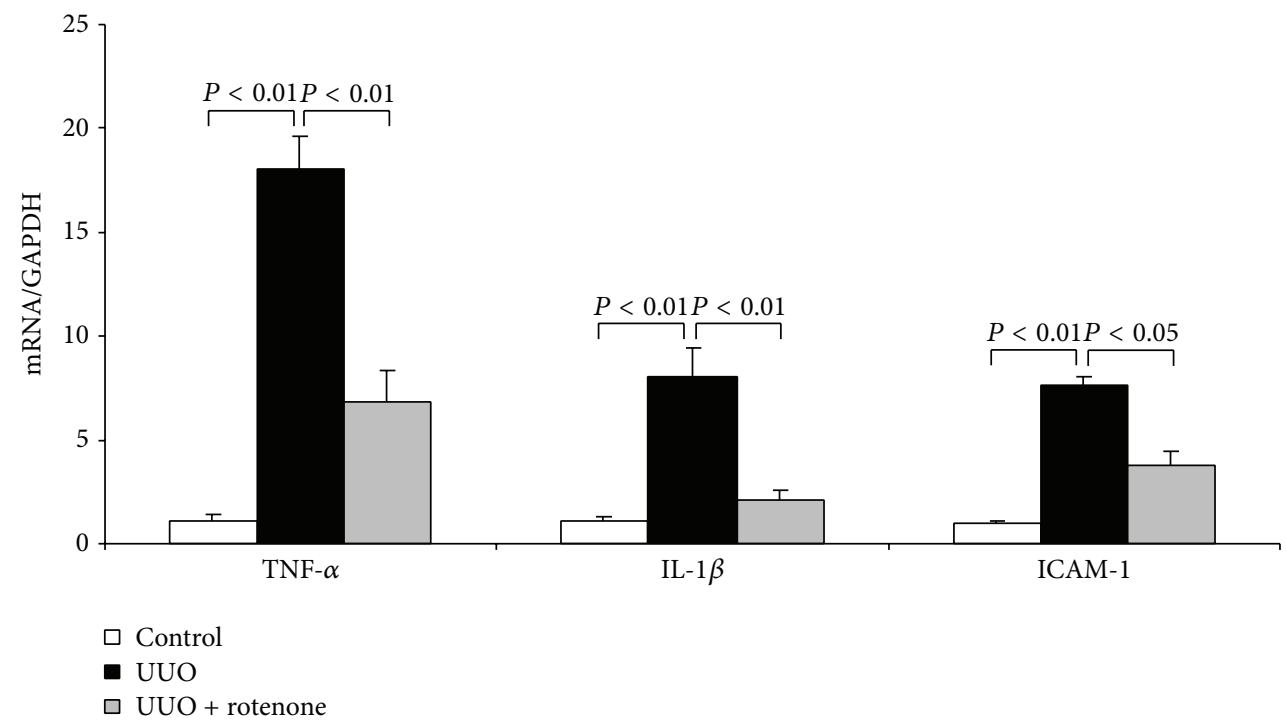

(a)

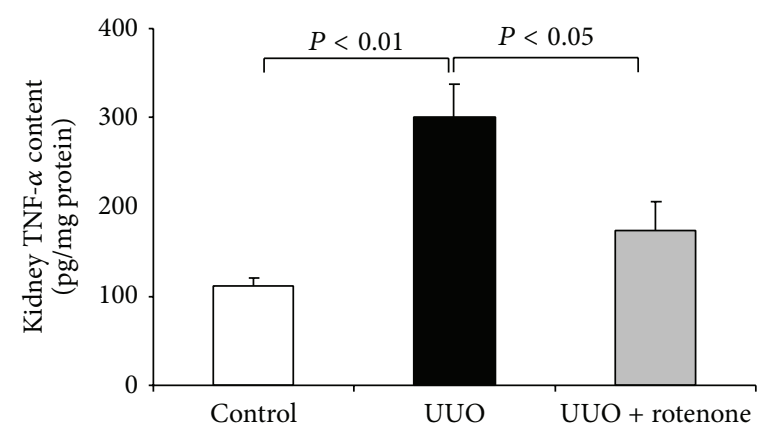

(b)

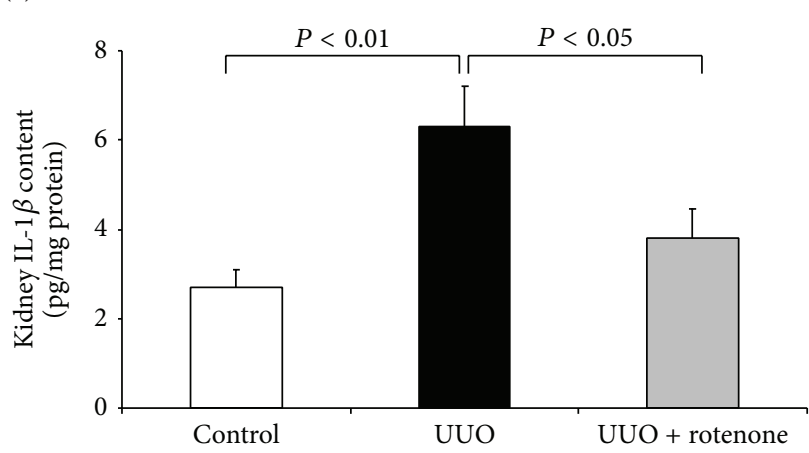

(c)

FIGURE 4: Rotenone treatment blunted inflammatory response in obstructed kidneys. (a) qRT-PCR analysis of TNF- $\alpha$, IL-1 $\beta$, and ICAM-1 mRNA expressions. (b) ELISA analysis of renal TNF- $\alpha$ protein level. (c) ELISA analysis of renal IL-1 $\beta$ protein level. $N=5$ in each group. Data are means $\pm \mathrm{SE}$.

Mitochondria are key cellular organelles in determining the cell fate. The dysfunction of mitochondria plays a pathogenic role in chronic heart failure $[24,25]$ and some central nervous diseases [26]. In kidney, recent reports indicated a potential involvement of mitochondrial dysfunction in mediating the development and progression of CKDs [1013]. In present study, we applied a mitochondrial complex I inhibitor to the mice subjected to UUO and observed a remarkable amelioration of kidney injury.

Mitochondrial damage not only results in the reduction of ATP level but also leads to the excessive production of ROS, which directly causes cellular injury and subsequently promotes mitochondrial dysfunction $[27,28]$. Because the mitochondrial abnormality is a known phenomenon in UUO kidneys $[10,12,29]$, in present study, we tested the efficacy of mitochondrial complex I inhibitor rotenone on the oxidative stress in obstructed kidneys and found that rotenone treatment strikingly reduced kidney TBARS content and HO-1 expression. These results demonstrated that inhibiting the activity of dysfunctional mitochondria definitely inhibited ROS overproduction, suggesting a critical role of mitochondrial dysfunction in mediating ROS overproduction in obstructed kidneys. More importantly, with the blockade of mitochondria-originated oxidative stress, the renal tubular injury was also significantly improved as determined by PAS staining, which suggested that mitochondrial oxidative stress served as a pathogenic factor in this pathological process.

Inflammation plays a detrimental role in the occurrence and development of kidney injury in various kidney diseases including CKDs. Both infiltrating inflammatory cells and renal resident cells contribute to inflammatory response in obstructive kidney disease [19, 30-32]. However, the detailed mechanisms leading to the inflammation in this model are still poorly understood. In present study, inhibition of mitochondrial complex I by rotenone led to a marked amelioration of inflammation as shown by significant suppression of proinflammatory cytokines, such as TNF- $\alpha$, IL$1 \beta$, and ICAM-1. These results suggested that mitochondrial dysfunction may play an important role in mediating the inflammatory response in obstructed kidneys. Also, a number of evidence demonstrated that oxidative stress could 

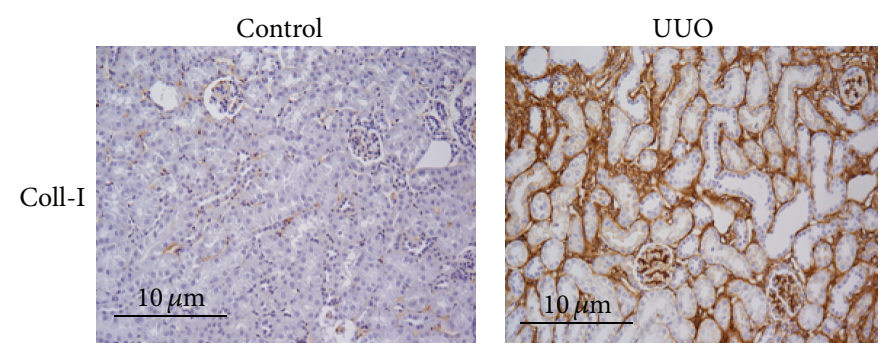

(a)
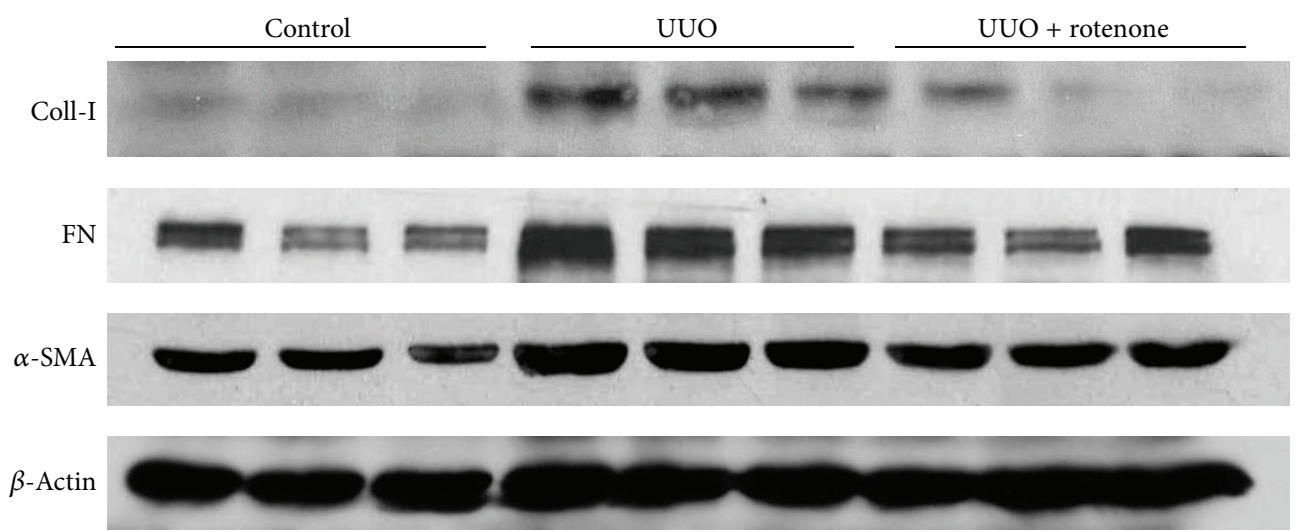

(b)

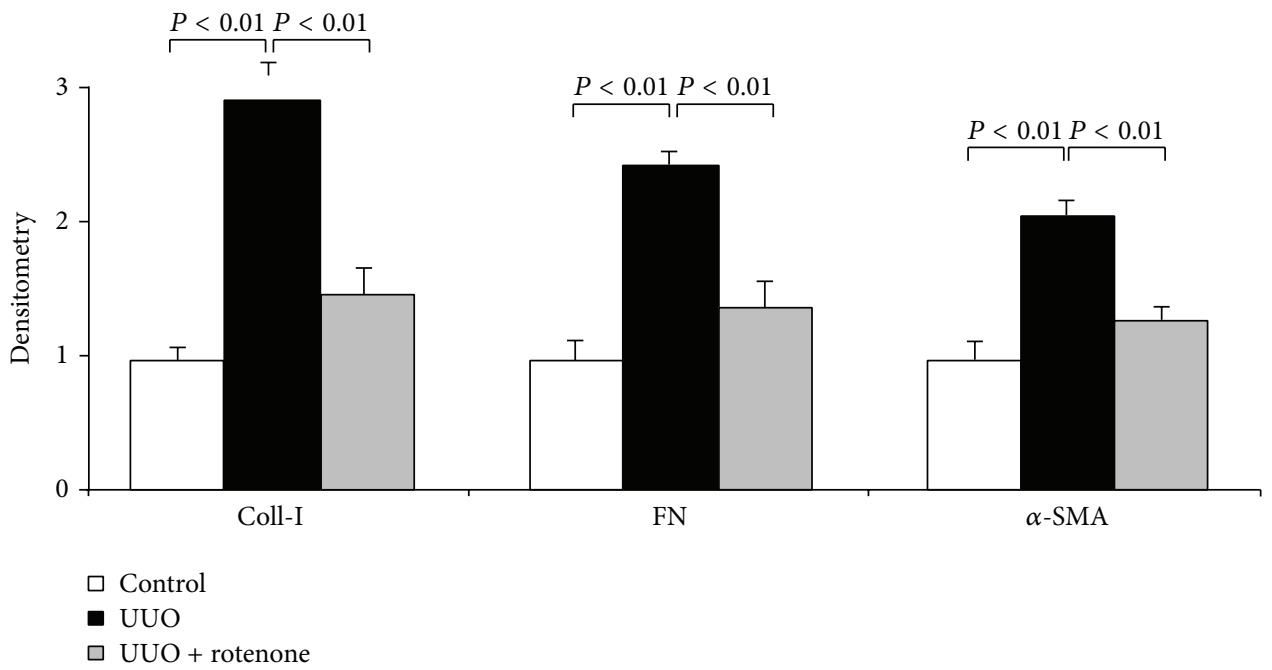

(c)

FIGURE 5: Rotenone treatment reduced protein expression of fibrotic markers in obstructed kidneys. (a) Immunohistochemistry of collagen I. (b) Fibrotic markers of collagen I, FN, and $\alpha$-SMA were determined by Western blotting, and $\beta$-actin was used as loading control. (c) Densitometry of Western blots. $N=5$ in each group. Data are means \pm SE.

trigger the inflammatory response in many pathological processes $[33,34]$. In present study, in line with the inhibition of oxidative stress by rotenone, the inflammatory response was also effectively blunted, indicating that mitochondriaderived oxidative stress may be a causative factor in triggering inflammation in obstructive kidney disease.

Fibrosis is a common outcome of all forms of renal injury. Fibrosis, as evidenced by the accumulation of extracellular matrix (ECM) [3] and cellular phenotypic alteration, was thought as a key player in the loss of renal function. Fibrosis not only is a result of various insults, such as inflammation and oxidative stress, but also contributes to the induction of inflammation and oxidative stress, forming a positive feedback loop. In agreement with this notion, blockade of mitochondrial oxidative stress and inflammation by rotenone was paralleled with the attenuation of fibrosis. To further investigate the potential mechanism of this antifibrotic action, we examined TGF- $\beta_{1}$, a key contributor of fibrosis in UUO [35, 36] and many other CDK models [37], and found that TGF$\beta_{1}$ was decreased by rotenone at both mRNA and protein 


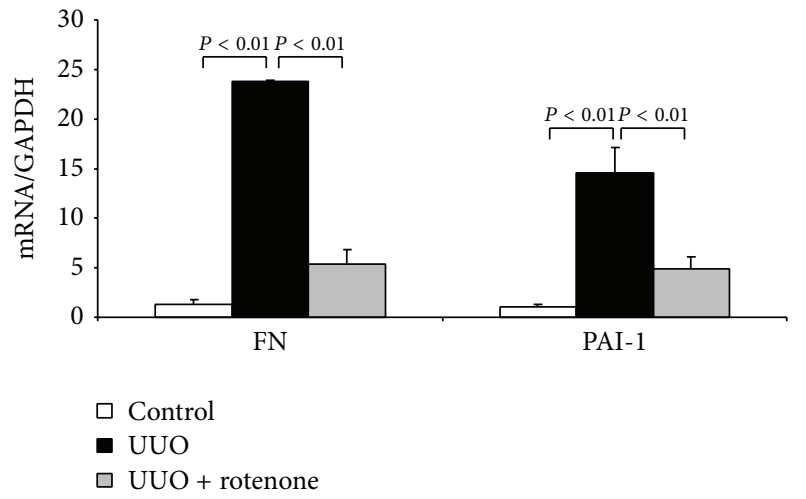

(a)

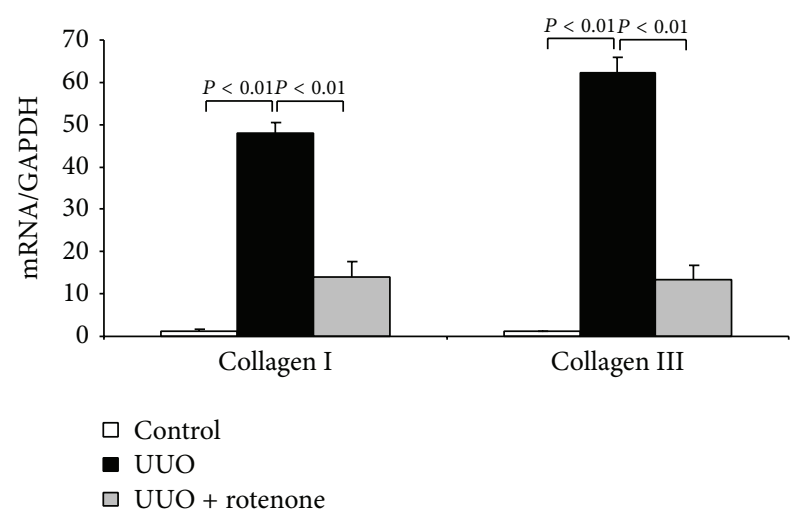

(b)

FIGURE 6: Rotenone treatment reduced mRNA expression of fibrotic markers in obstructed kidneys. (a) qRT-PCR analysis of FN and PAI-1 mRNA levels. (b) qRT-PCR analysis of collagen I and collagen III mRNA levels. $N=5$ in each group. Data are means \pm SE.

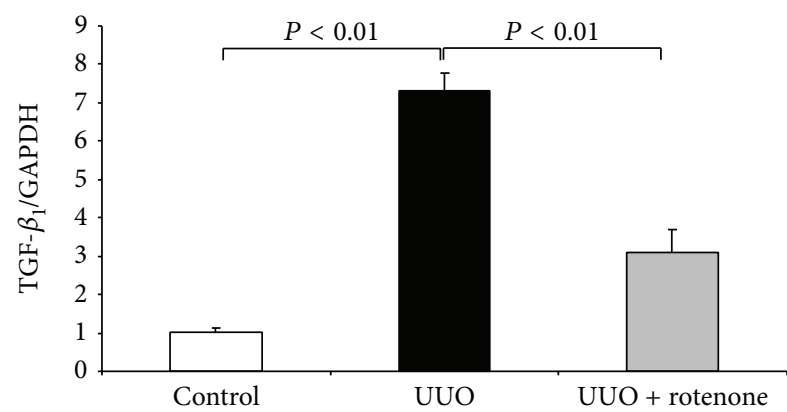

(a)

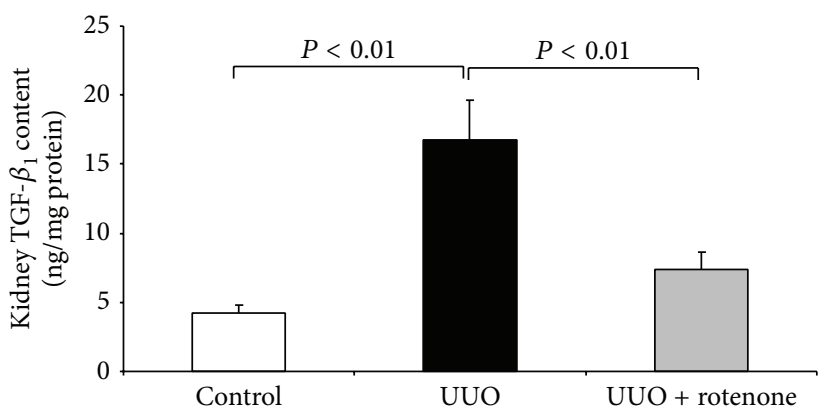

(b)

FIGURE 7: Rotenone treatment decreased renal TGF- $\beta_{1}$ induction in obstructed kidneys. (a) qRT-PCR analysis of renal TGF- $\beta_{1}$ mRNA expression. (b) ELISA analysis of renal TGF- $\beta_{1}$ protein expression. $N=5$ in each group. Data are means \pm SE.

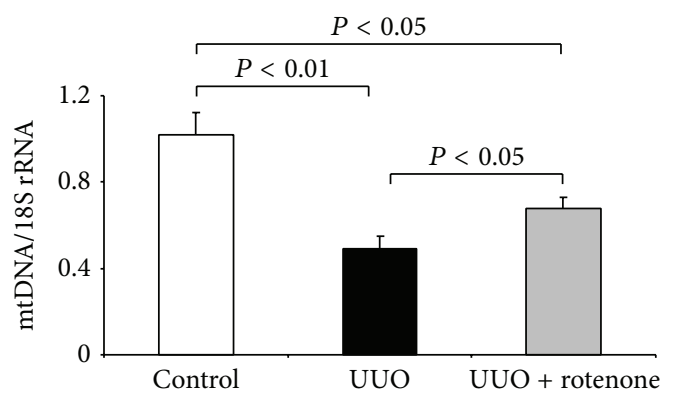

(a)

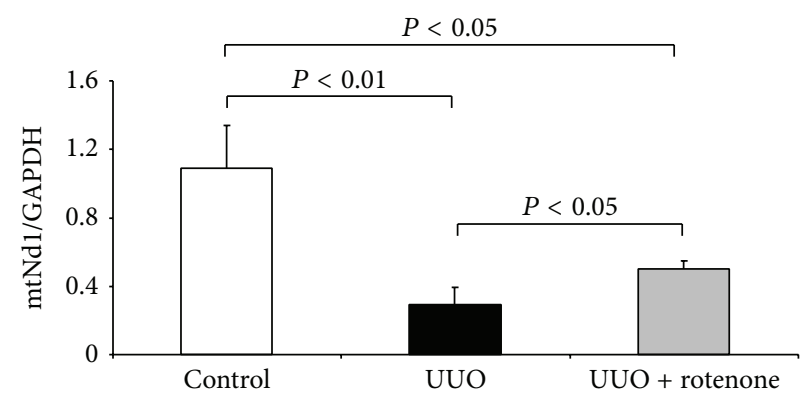

(b)

FIGURE 8: Rotenone treatment partially reversed the reduction of mtDNA copy number and mtND1 expression in obstructed kidneys. (a) mtDNA copy number was determined by qRT-PCR. (b) mtND1 expression was determined by qRT-PCR. $N=5$ in each group. Data are means \pm SE.

levels. These data suggested that the antifibrotic action of rotenone is possibly through its blockade of TGF- $\beta_{1}$, at least to some extent. Reduction of TGF- $\beta_{1}$ could be secondary to the attenuation of oxidative stress and inflammation because both ROS [38] and inflammatory cytokines [39] have been shown to be responsible for the TGF- $\beta_{1}$ induction.
In summary, present study demonstrated that inhibiting the activity of dysfunctional mitochondria in obstructed kidney by a mitochondrial complex I inhibitor significantly attenuated kidney injury in parallel with the blockade of oxidative stress, inflammation, and fibrotic response. These results suggested a causative role of mitochondrial 
dysfunction in mediating the obstructive kidney injury. And a pathogenic loop formed by mitochondrial oxidative stress, inflammation, and fibrosis may exist in this pathological process. Targeting mitochondrial dysfunction may serve as a novel therapeutic strategy for the treatment of obstructive kidney disease and other CKDs.

\section{Conflict of Interests}

There is no conflict of interests to disclose.

\section{Authors' Contribution}

Ying Sun and Yue Zhang contributed equally to this work.

\section{Acknowledgments}

This work was supported by Grants from National Natural Science Foundation of China (nos. 81370802 and 81300591), National Basic Research Program of China (973 Program) (no. 2012CB517602), and the Natural Science Foundation of Jiangsu Province (no. BK2012001).

\section{References}

[1] A. A. Eddy, "Can renal fibrosis be reversed?" Pediatric Nephrology, vol. 20, no. 10, pp. 1369-1375, 2005.

[2] M. El Nahas, "The global challenge of chronic kidney disease," Kidney International, vol. 68, no. 6, pp. 2918-2929, 2005.

[3] A. A. Eddy, "Molecular basis of renal fibrosis," Pediatric Nephrology, vol. 15, no. 3-4, pp. 290-301, 2000.

[4] G. Zheng, J. G. Lyons, K. T. Thian et al., "Disruption of E-cadherin by matrix metalloproteinase directly mediates epithelial-mesenchymal transition downstream of transforming growth factor- $\beta 1$ in renal tubular epithelial cells," American Journal of Pathology, vol. 175, no. 2, pp. 580-591, 2009.

[5] K. Inazaki, Y. Kanamaru, Y. Kojima et al., "Smad3 deficiency attenuates renal fibrosis, inflammation, and apoptosis after unilateral ureteral obstruction," Kidney International, vol. 66, no. 2, pp. 597-604, 2004.

[6] M. Madesh and G. Hajnóczky, "VDAC-dependent permeabilization of the outer mitochondrial membrane by superoxide induces rapid and massive cytochrome c release," Journal of Cell Biology, vol. 155, no. 6, pp. 1003-1015, 2001.

[7] P. Xie, L. Sun, B. Nayak et al., "C/EBP- $\beta$ modulates transcription of tubulointerstitial nephritis antigen in obstructive uropathy," Journal of the American Society of Nephrology, vol. 20, no. 4, pp. 807-819, 2009.

[8] J. Rojas-Rivera, A. Ortiz, and J. Egido, "Antioxidants in kidney diseases: the impact of bardoxolone methyl," International Journal of Nephrology, vol. 2012, Article ID 321714, 11 pages, 2012.

[9] D. Impellizzeri, E. Esposito, J. Attley, and S. Cuzzocrea, “Targeting inflammation: new therapeutic approaches in chronic kidney disease (CKD)," Pharmacological Research, vol. 81, pp. 91-102, 2014.

[10] I. M. García, L. Altamirano, L. Mazzei et al., "Role of mitochondria in paricalcitol-mediated cytoprotection during obstructive nephropathy," The American Journal of Physiology-Renal Physiology, vol. 302, no. 12, pp. F1595-F1605, 2012.
[11] D. M. Small, J. S. Coombes, N. Bennett, D. W. Johnson, and G. C. Gobe, "Oxidative stress, anti-oxidant therapies and chronic kidney disease," Nephrology, vol. 17, no. 4, pp. 311-321, 2012.

[12] Y. Xu, S. Ruan, X. Wu, H. Chen, K. Zheng, and B. Fu, "Autophagy and apoptosis in tubular cells following unilateral ureteral obstruction are associated with mitochondrial oxidative stress," International Journal of Molecular Medicine, vol. 31, no. 3, pp. 628-636, 2013.

[13] J. F. Chen, H. Liu, H. F. Ni et al., "Improved mitochondrial function underlies the protective effect of pirfenidone against tubulointerstitial fibrosis in 5/6 nephrectomized rats," PLoS ONE, vol. 8, no. 12, Article ID e83593, 2013.

[14] R. Dhingra and L. A. Kirshenbaum, "Regulation of mitochondrial dynamics and cell fate," Circulation Journal, vol. 78, pp. 803-810, 2014.

[15] A. Devin and M. Rigoulet, "Mechanisms of mitochondrial response to variations in energy demand in eukaryotic cells," The American Journal of Physiology-Cell Physiology, vol. 292, no. 1, pp. C52-C58, 2007.

[16] S. Michel, A. Wanet, A. de Pauw, G. Rommelaere, T. Arnould, and P. Renard, "Crosstalk between mitochondrial (dys)function and mitochondrial abundance," Journal of Cellular Physiology, vol. 227, no. 6, pp. 2297-2310, 2012.

[17] E. M. V. de Cavanagh, F. Inserra, M. Ferder, and L. Ferder, "From mitochondria to disease: role of the renin-angiotensin system," American Journal of Nephrology, vol. 27, no. 6, pp. 545-553, 2007.

[18] M. Su, A. Dhoopun, Y. Yuan et al., "Mitochondrial dysfunction is an early event in aldosterone-induced podocyte injury," The American Journal of Physiology: Renal Physiology, vol. 305, no. 4, pp. F520-F531, 2013.

[19] R. L. Chevalier, M. S. Forbes, and B. A. Thornhill, "Ureteral obstruction as a model of renal interstitial fibrosis and obstructive nephropathy," Kidney International, vol. 75, no. 11, pp. 11451152, 2009.

[20] K. M. A. Rouschop, M. E. Sewnath, N. Claessen et al., "CD44 deficiency increases tubular damage but reduces renal fibrosis in obstructive nephropathy," Journal of the American Society of Nephrology, vol. 15, no. 3, pp. 674-686, 2004.

[21] Z. Jia, H. Wang, and T. Yang, "Microsomal prostaglandin E synthase 1 deletion retards renal disease progression but exacerbates anemia in mice with renal mass reduction," Hypertension, vol. 59, no. 1, pp. 122-128, 2012.

[22] S. S. Badal and F. R. Danesh, "New insights into molecular mechanisms of diabetic kidney disease," American Journal of Kidney Diseases, vol. 63, pp. S63-S83, 2014.

[23] M. E. Hall, J. M. do Carmo, A. A. da Silva, L. A. Juncos, Z. Wang, and J. E. Hall, "Obesity, hypertension, and chronic kidney disease," International Journal of Nephrology and Renovascular Disease, vol. 7, pp. 75-88, 2014.

[24] D. B. Zorov, C. R. Filburn, L. O. Klotz, J. L. Zweier, and S. J. Sollott, "Reactive oxygen species (ROS)-induced ROS release: a new phenomenon accompanying induction of the mitochondrial permeability transition in cardiac myocytes," Journal of Experimental Medicine, vol. 192, no. 7, pp. 1001-1014, 2000.

[25] Y. R. Chen and J. L. Zweier, "Cardiac mitochondria and reactive oxygen species generation," Circulation Research, vol. 114, no. 3, pp. 524-537, 2014.

[26] M. E. Witte, D. J. Mahad, H. Lassmann, and J. van Horssen, "Mitochondrial dysfunction contributes to neurodegeneration in multiple sclerosis," Trends in Molecular Medicine, vol. 20, pp. 179-187, 2014. 
[27] D. C. Wallace, "Mitochondrial diseases in man and mouse," Science, vol. 283, no. 5407, pp. 1482-1488, 1999.

[28] D. C. Wallace, "A mitochondrial paradigm of metabolic and degenerative diseases, aging, and cancer: a dawn for evolutionary medicine," Annual Review of Genetics, vol. 39, pp. 359-407, 2005.

[29] M. S. Forbes, B. A. Thornhill, C. I. Galarreta, J. J. Minor, K. A. Gordon, and R. L. Chevalier, "Chronic unilateral ureteral obstruction in the neonatal mouse delays maturation of both kidneys and leads to late formation of atubular glomeruli," American Journal of Physiology: Renal Physiology, vol. 305, pp. F1736-F1746, 2013.

[30] G. F. Schreiner, K. P. G. Harris, M. L. Purkerson, and S. Klahr, "Immunological aspects of acute ureteral obstruction: immune cell infiltrate in the kidney," Kidney International, vol. 34, no. 4, pp. 487-493, 1988.

[31] Y. Le Meur, G. H. Tesch, P. A. Hill et al., "Macrophage accumulation at a site of renal inflammation is dependent on the M-CSF/c-fms pathway," Journal of Leukocyte Biology, vol. 72, no. 3, pp. 530-537, 2002.

[32] D. M. Lenda, E. Kikawada, E. R. Stanley, and V. R. Kelley, "Reduced macrophage recruitment, proliferation, and activation in colony-stimulating factor-1-deficient mice results in decreased tubular apoptosis during renal inflammation," Journal of Immunology, vol. 170, no. 6, pp. 3254-3262, 2003.

[33] J. M. Vieira Jr., E. Mantovani, L. Tavares Rodrigues et al., "Simvastatin attenuates renal inflammation, tubular transdifferentiation and interstitial fibrosis in rats with unilateral ureteral obstruction," Nephrology Dialysis Transplantation, vol. 20, no. 8, pp. 1582-1591, 2005.

[34] Y. Li, R. F. Schwabe, T. DeVries-Seimon et al., "Free cholesterolloaded macrophages are an abundant source of tumor necrosis factor- $\alpha$ and interleukin- 6 : model of NF- $\kappa$ B- and map kinasedependent inflammation in advanced atherosclerosis," The Journal of Biological Chemistry, vol. 280, no. 23, pp. 21763-21772, 2005.

[35] S. Klahr and J. Morrissey, "Obstructive nephropathy and renal fibrosis: The role of bone morphogenic protein-7 and hepatocyte growth factor," Kidney International, Supplement, vol. 64, no. 87, pp. S105-S112, 2003.

[36] M. Sato, Y. Muragaki, S. Saika, A. B. Roberts, and A. Ooshima, "Targeted disruption of TGF- $\beta 1 / \mathrm{Smad} 3$ signaling protects against renal tubulointerstitial fibrosis induced by unilateral ureteral obstruction," Journal of Clinical Investigation, vol. 112, no. 10, pp. 1486-1494, 2003.

[37] F. J. López-Hernández and J. M. López-Novoa, "Role of TGF- $\beta$ in chronic kidney disease: an integration of tubular, glomerular and vascular effects," Cell and Tissue Research, vol. 347, no. 1, pp. 141-154, 2012.

[38] H. B. Lee, M. R. Yu, Y. Yang, Z. Jiang, and H. Ha, "Reactive oxygen species-regulated signaling pathways in diabetic nephropathy," Journal of the American Society of Nephrology, vol. 14, no. 3, pp. S241-S245, 2003.

[39] D. E. Sullivan, M. Ferris, H. Nguyen, E. Abboud, and A. R. Brody, "TNF- $\alpha$ induces TGF- $\beta 1$ expression in lung fibroblasts at the transcriptional level via AP-1 activation," Journal of Cellular and Molecular Medicine, vol. 13, no. 8 B, pp. 1866-1876, 2009. 


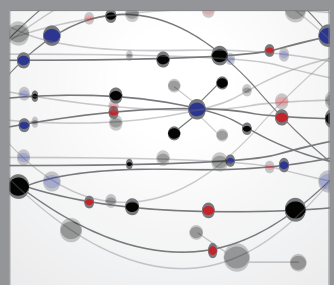

The Scientific World Journal
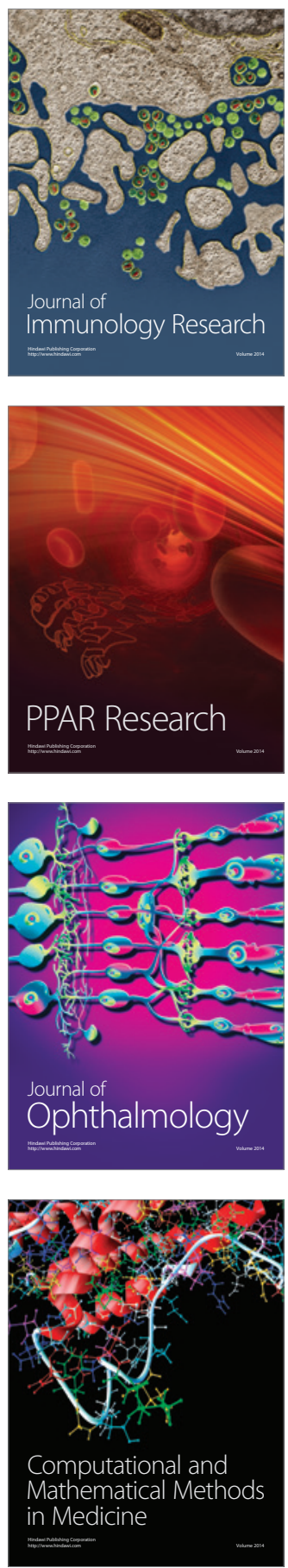

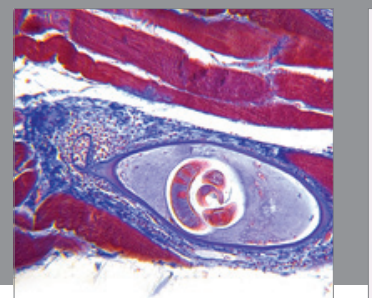

Gastroenterology

Research and Practice
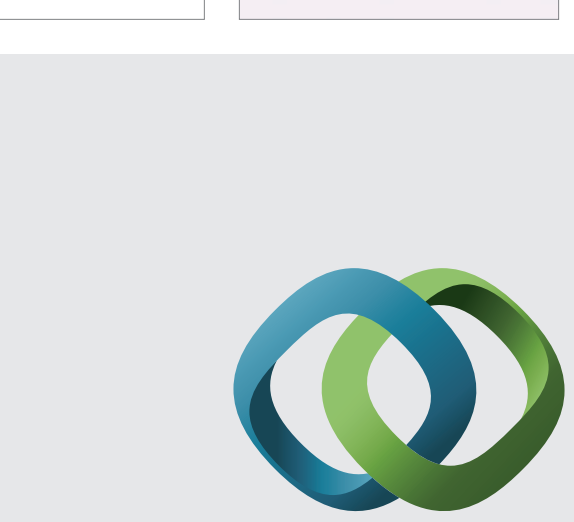

\section{Hindawi}

Submit your manuscripts at

http://www.hindawi.com
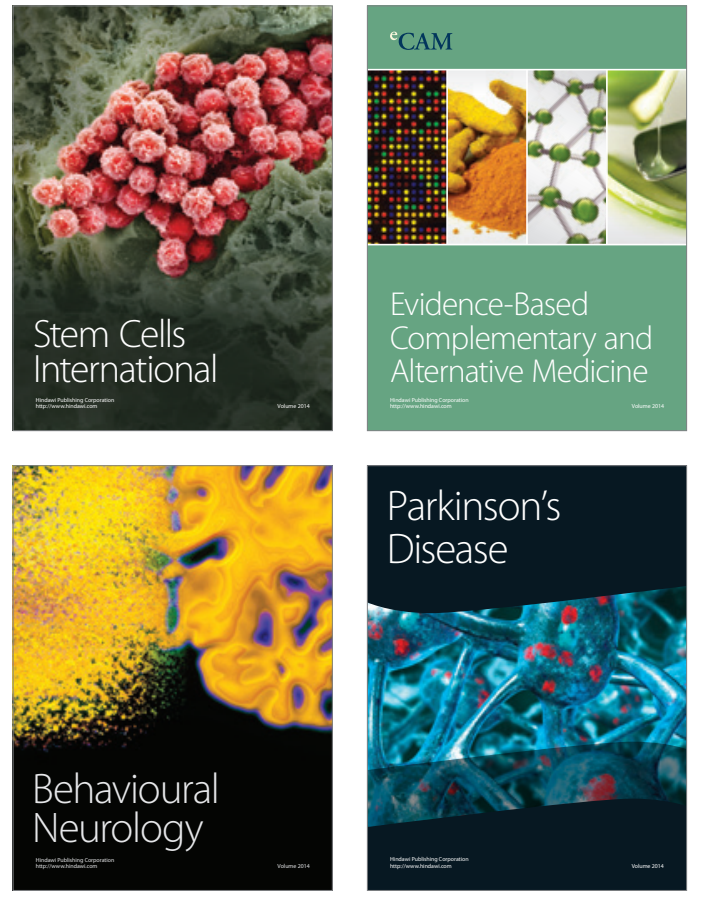
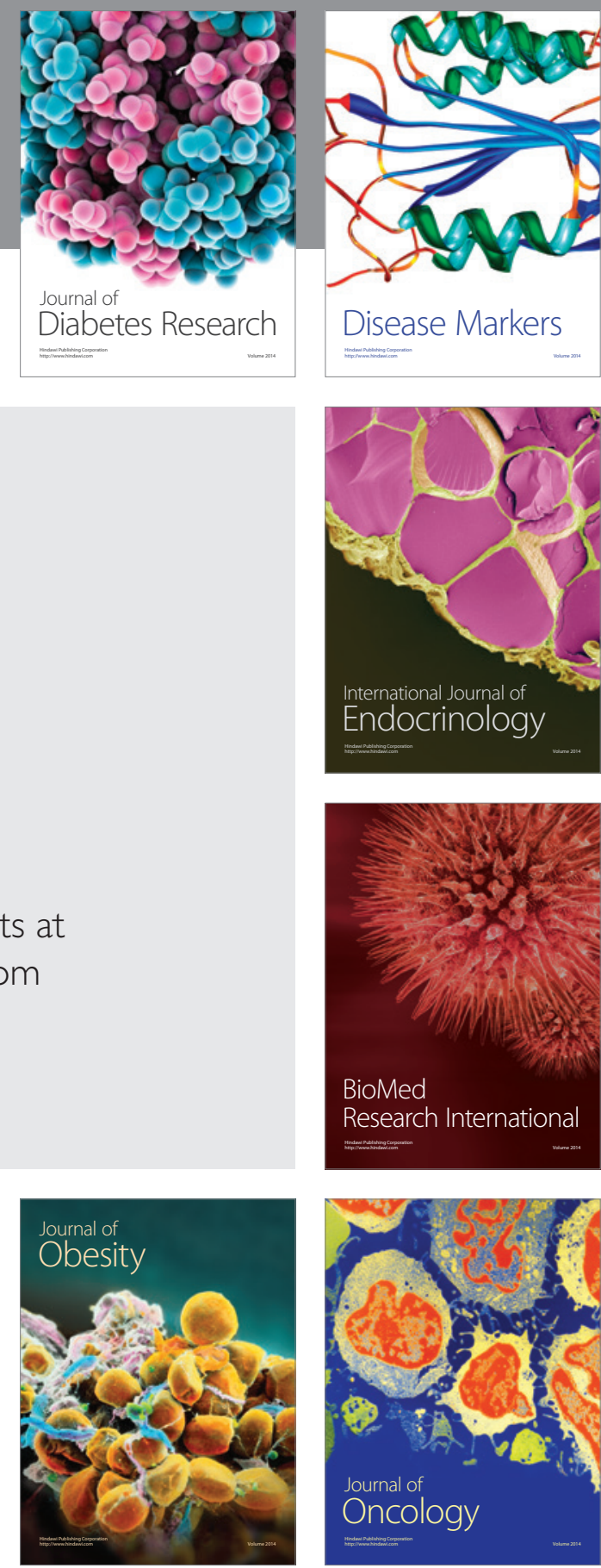

Disease Markers
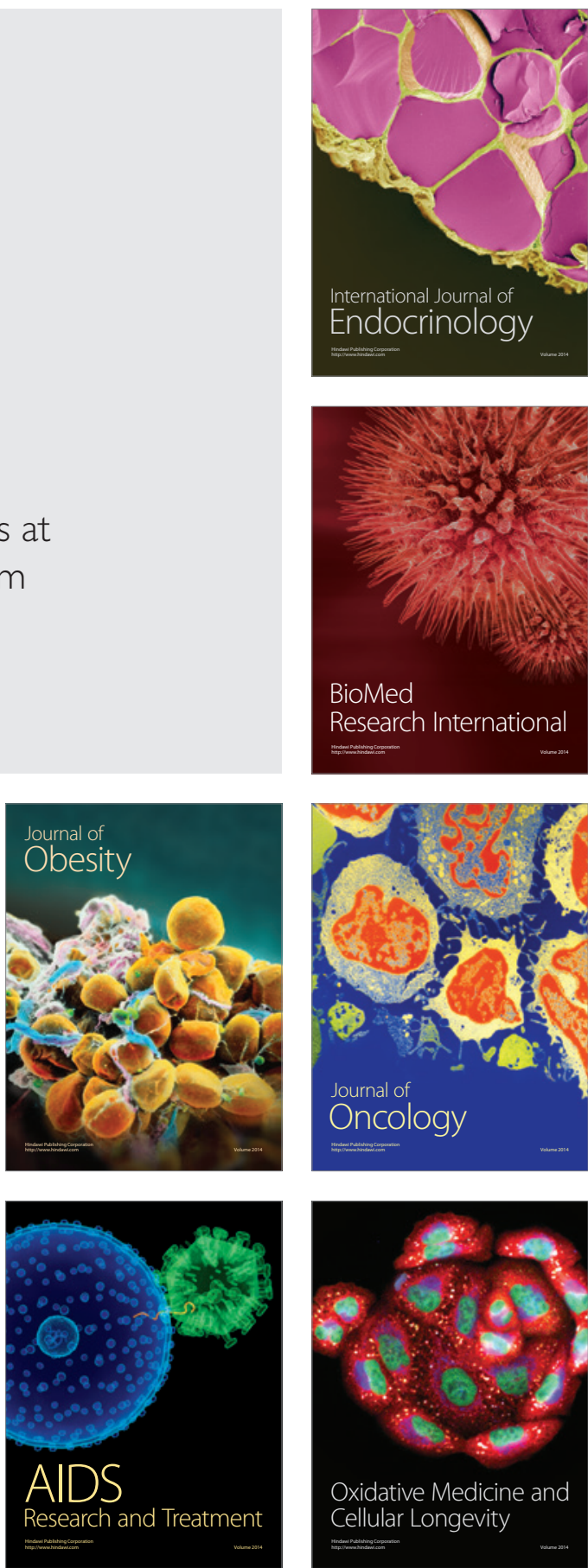